\title{
Téoros
}

Revue de recherche en tourisme

\section{L'intégration du patrimoine régional aux activités touristiques}

\section{Cornéliu Kirjan}

Volume 7, numéro 1, mars 1988

Cultures régionales et tourisme

URI : https://id.erudit.org/iderudit/1080430ar

DOI : https://doi.org/10.7202/1080430ar

Aller au sommaire du numéro

Éditeur(s)

Université du Québec à Montréal

ISSN

0712-8657 (imprimé)

1923-2705 (numérique)

Découvrir la revue

Citer cet article

Kirjan, C. (1988). L'intégration du patrimoine régional aux activités touristiques. Téoros, 7(1), 28-29. https://doi.org/10.7202/1080430ar d'utilisation que vous pouvez consulter en ligne.

https://apropos.erudit.org/fr/usagers/politique-dutilisation/ 


\section{L'intégration du patrimoine régional aux activités touristiques}

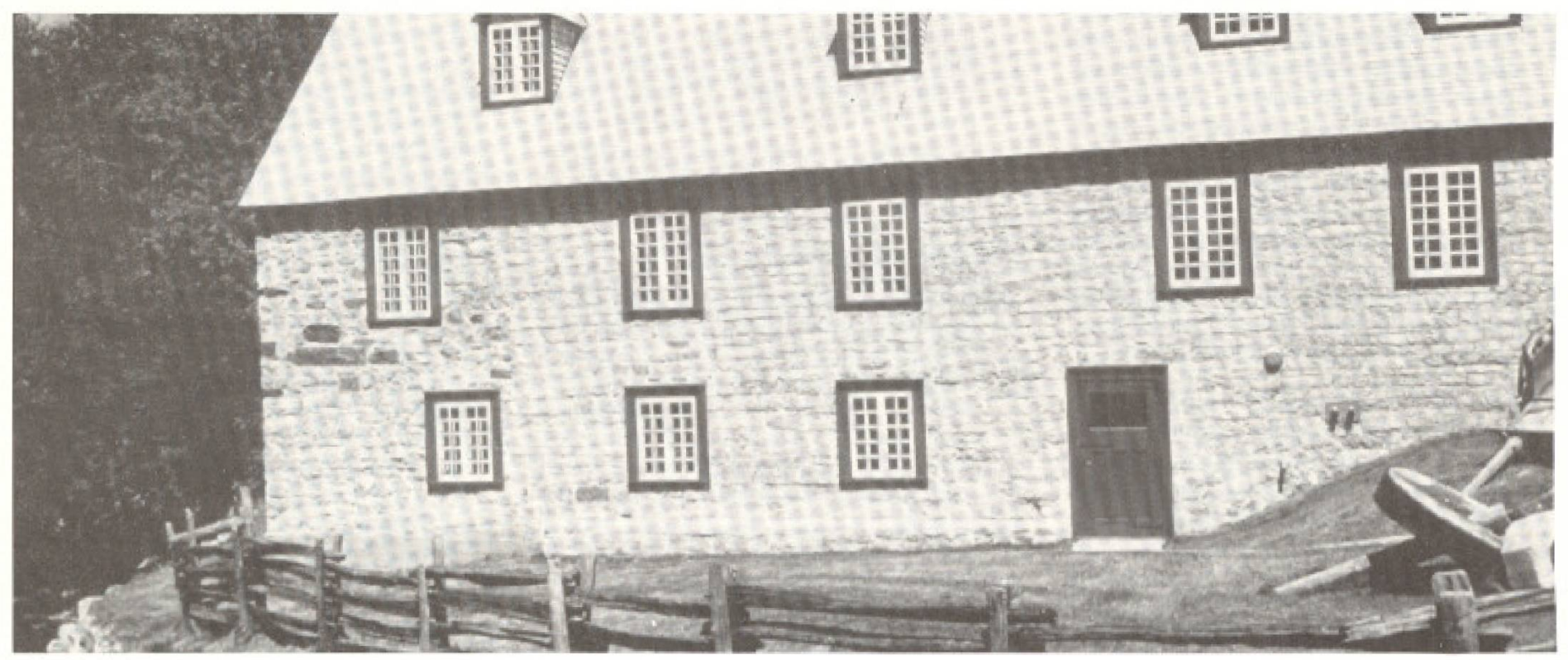

"Le voyage est une porte par où l'on sort de la réalité comme pour pénétrer dans une réalité inexplorée qui semble un rêve."

Maupassant

Durant les dernières années, de nombreux sondages et études ont démontré clairement que le patrimoine culturel d'un pays ou d"une région constitue un des principaux facteurs d'attraction du tourisme actuel. L'importance du patrimoine culturel comme facteur d'attraction touristique est tellement grande qu'on emploie de plus en plus le terme de "tourisme culturel" pour désigner ce phénomène qui prend beaucoup d'ampleur. Le contact avec une culture spécifique, différente de la sienne, constitue une des moti- vations importantes de ceux qui pour une certaine periode de l'année se transforment de personnes ordinaires en touristes.

Dans ce sens, la spécificité culturelle du Québèc et de ses régions constitue un élément majeur d'attraction touristique. En dépit de cet axiome, on est obligé de reconnaître qu'au Québec les résultats laissent à désirer et que le patrimoine régional est peu intégré au developpement touristique.

Il faut mentionner que la notion de patrimoine régional tellè qu'employée ne fait aucune référence à une question de valeur (régional versus national) mais qu'clle est employée seulement dans un sens d'appartenance territoriale (le patrimoine qui se trouve sur le territoire d'une région).

Mais de quel patrimoine s'agit-il? Cette ques= tion est d'autant plus pertinente depuis qu'on sait que l'évolution de la notion de patrimoine a provoqué des changements majeurs en ce qui concerne les formes et les moyens de mise en valeur du patrimoine. Et de plus, e'est le choix judicieux de la forme et des moyens de mise en valeur qui constituent la base de succès dans l'intégration du patrimoine régional au développement touristique.

"Tous les pays qui $n$ 'ont plas de légende seront condamués à mourir."

La Tour du Pin Chambly
Aujourd hui, la notion de patrimoine englobe tout ce qui peut être transmis d'une génération à une autre à l'intérieur d'une communauté sur un territoire donné. Dans son évolution, la notion de patrimoine est passée de patrimoine-objet, sectoriel, à patrimoine-identité, global. Si le patrimoineobjet était facilement définissable dans le cas du patrimoine-identité ou patrimoine global , on peut parler plutôt de paramètres qui le caractérisent: temps, espace, environnement naturel, dynamique et évolution sociales, oeuvres (matérielles et spirituelles).

Cette évolution du patrimoine-objet au patrimoine-identité a été accompagnée de modifications importantes quant à l'étude et la mise en valeur. Le patrimoine-identité, de plus en plus large et complexe, ne pouvait plus être étudié par un seul spécialiste ou par des spécialistes isolés. On assiste à l'apparition de l'étude interdisciplinaire, seul moyen de saisir les relations existantes entre les diverses composantes de ce patrimoine élargi.

On constate aussi l'apparition de nouvelles formes de mise en valeur du patrimoine qui se devaient de correspondre aux nouvelles réalités patrimoniales. Le musée classique axé sur les collections d'artefacts n"est plus la seule forme de mise en valeur. Dans son évolution, la notion de patrimoine génère de nouvelles formes: musée en air libre, centre d'interprétation, circuit patrimonial et culturel, musée de voisinage, ecomusée. 
Le musée dit "classique", représente la forme de mise en valeur qui correspondait à une certaine ćtape de développement de la société ainsi qu'à une certaine notion de patrimoine - le patrimoine-objet - qu'il fallait (avec raison d'ailleurs) protéger. Il correspond à une période de découverte du patrimoine et comme beaucoup d'autres découvertes, le patrimoine a commencé à être connu par ses aspects matériels et de manière disparate. Mais le musée lui-même a evolué et aujourd'hui, à l'exception du musée d'art, le concept de musée universel est presque disparu. Chaque pays, chaque région développe des formes spécifiques en suivant l'évolution et les interrogations que la société qui le génère se posent. Le rồle mẻme du musée a évolué de muséedélectation au musée-instrument de développement culturel et d'éducation.

L'apparition des centres d'interprétation après la Deuxième Guerre mondiale illustre bien la relation existante entre l'évolution de la notion du patrimoine, les formes de mise en valeur et le tourisme. Les divers éléments du patrimoine sont interprétés dans leur contexte, les objets ne sont plus collectionnés seulement pour les préserver et les entreposer mais spécialement pour illustrer un thème choisi. Divers moyens audio-visuels sont employés pour interpréter "le patrimoine" sur le site mềme ou il a été découvert. L'interprétation se fait par des équipes multidisciplinaires et elle vise non pas les objets mais les relations qui existent entre les objets eux-mêmes ainsi qu'entre les objets et les personnes qui les ont produit ou utilisé.

Enfin, l'évolution de la notion du patrimoine trouve aujourd'hui son accomplissement dans le patrimoine-identité et dans l'écomusée comme forme de mise en valeur correspondante. Cette fois, ce qu'on peut présenter et mettre en valeur c'est avant tout le vécu et le savoir faire d'une population dans une région donnée.

L'écomusée est la forme de mise en valeur qui regarde non pas des objets ou un lieu mais représente l'interprétation et la valorisation d'un territoire et d'une population avec tout son savoir faire.

D'autres formes de mise en valeur du patrimoine tel le circuit patrimonial et l'itinéraire culturel jouent un rôle important dans l'intégration du patrimoine régional au développement touristique. De plus, leur rỏle est très important pour le milieu local car ils permettent une prise de conscience globale et rapide des valeurs locales.

"Car ce n'est pas assez d'avoir l'esprit bon
mais le principal est de l'appliquer bien." Descartes

Visiter un site historique, découvrir la richesse culturelle d'une région et le savoir faire $d^{+} u n e$ population nous permettent à la fois une connaissance des autres et de nousmêmes. Nombre d'études et de statistiques ont déjà démontré que le patrimoine constitue un des principaux facteurs d'attraction du tourisme actuel. Mais de quelle manière on peut intégrer le patrimoine régional au développement touristique? La mise en valeur du patrimoine régional et son intégration au développement touristique doivent satisfaire à plusieur's conditions dont quatre nous semblent essentielles: authenticité et souci de qualité, polyvalence, orientation éducative et collaboration des partenaires impliqués dans le processus.

\section{Authenticité et souci de qualité.}

Le patrimoine régional québécois deviendra un atout réel seulement quand les visiteurstouristes réaliseront que la visite d'une région constitue une entrée dans un monde d'un type particulier, différent de leur monde quotidien. Trop souvent par le passé des projets ont ćté échafaudés en partant des idées ou des exemples importés d'ailleurs. On oubliait trop souvent que les exemples choisis avalient été générés par une autre population ayant un autre vécu et dans un contexte différent.

L'authenticité et le souci de qualité dans. l'interprétation du patrimoine régional génèrent une dynamique à double sens dans la relation visiteur-visité. Si le visiteur-touriste est attiré par ce monde authentique qui n'est pas le sien, il contribuera à son tour à consolider le sentiment d'appartenance et de fierté du visité. De son cóté dans sa recherche d'authenticité et de qualité, le visité tout en découvrant la richesse de son patrimoine se découvrira lui-même.

Pour réaliser cette dynamique, on doit nécessairement passer par l'étape d'acquisition de connaissances qui permettra à une région de découvrir le potentiel qu'elle possède, d'identifier les ressources et par comparaison de prendre connaissance des différences qui lui permettront de se définir.

Dans ce processus, la participation de la population est essentielle et dans ce sens, on doit mentionner que lá formule de l'écomusée est la plus adéquate pour favoriser cette participation.

\section{Polyvalence.}

La polyvalence concerne aussi bien les divers aspects du patrimoine que les activités et les equipements offerts aux touristes. Pour pouvoir donner aux visiteurs une image de patrimoine-identité d'une région, on se doit de mettre en valeur, dans la mesure du possible, l'ensemble des éléments qui constitue le patrimoine-identité. Dans ce sens, on doit mentionner que certaines formes de mise en valeur du patrimoine - écomusée, circuits patrimoniaux ct culturels, centre d'interprétation - offrent à priori cette polyvalence (diversité des eléments patrimoniaux, diversité d'activités vivantes et d'équipements).

Ces formes de mise en valeur permettent aux touristes de prendre connaissance de la richesse patrimoniale d'une région sans pour autant les concentrer dans un seul endroit. De plus, ces formes de mise en valeur per- mettent aux touristes de mieux comprendre la relation étroite qui existe entre l'environnement naturel de chaque région et la population qui, en s'adaptant, a façonné cet environnement à sa manière.

\section{Aspect éducatif.}

L'aspect éducatif doit être toujours présent et de qualité supérieure. Il doit viser aussi bien le touriste que la population locale qui reçoit, pour lui permettre à la fois de découvrir et de se découvrir. Une importance accrue doit être accordese aux moyens d'interprétation qui visent spécifiquement la clientèle scolaire. Dans ce sens, les concepts d'interprétation du patrimoine doivent développer des moyens de communication qui visent aussi bien les adultes que les jeunes.

\section{Collaboration}

La collaboration des divers intervenants constitue une des conditions essentielles pour une bonne intégration du patrimoine régional au développement touristique. Cette collaboration implique spécifiquement trois groupes principaux: la population, les agents de tourisme (publics et privés) et les agents de connaissance. L'implication et la collaboration de la population peut s'avérer déterminant pour tout projet qui vise la mise en valeur du patrimoine régional et son intégration au développement touristique. Une population non impliquéc aura comme résul= tat un manque de participation, des réactions négatives envers les touristes ou une surexploitation de ceux-ci. L'integration doit se faire d'une maniere qui permettra à la population locale de continuer à vivre normalement dans un milieu qu'elle est fière de montrer et partager avec les visiteurs.

La collaboration des agents de tourisme (publics et privés) avec les agents de connaissance (spécialistes de patrimoine) garantit aux touristes un produit authentique, bien documenté et de qualité. Il faut dire que cette collaboration a souvent fait défaut par le passé. Elle doit se manifester aussi bien dans la réalisation d'un projet qu'au niveau de l'information destinée aux touristes. On constate souvent que ce manque de collaboration a comme résultat des moyens d'information (brochures, guides, cartes touristiques) dans lesquels le patrimoine régional est mal présenté ou carrément absent.

Enfin, une plus grande collaboration entre les divers organismes gouvernementaux qui touchent au tourisme et au patrimoine ainsi qu'entre ceux-ci et les organismes régionaux et locaux ne fera que contribuer à une meilleure intégration du patrimoine régional au développement touristique.

Pour finir, on doit mentionner qu'en dépit des apparenoes, l'industrie touristique est une industrie polluante. Dans le cadre des projets qui visent l'intégration du patrimoine régional au développement touristique, une attention particulière devra être accordée aux répercussions que la pollution (visuelle et physique) peut avoir sur notre cadre de vie. $f$ 